\title{
The Expanding Universe*
}

\section{By Sir Arthur Eddington, F.R.S.}

$\mathrm{I}^{\mathrm{N}}$ recent years the line-of-sight velocities of about ninety of the spiral nebulæ have been measured. The distances of some of the nearest of them have been determined by a fairly trustworthy method, and for others rude estimates depending on statistical methods are available. When we survey these data, a remarkable state of affairs is revealed. The spiral nebulæ are almost unanimously running away from us; moreover, the greater the distance the greater the speed of recession. The law of increase is found to be fairly regular, the speed being simply proportional to the distance. The progression has been traced up to a distance of more than 100 million light-years, where the recession is $20,000 \mathrm{~km}$. per sec.-about the speed of an $\alpha$-particle.

At first sight this looks as though the spiral nebulæ had a rather pointed aversion to our society; but a little consideration will show that the phenomenon is merely a uniform dilation of the system and is not specially directed at us. If this room were suddenly to expand to twice its present size, the seats separating in proportion, you would notice that everyone in the room had moved away from you. Your neighbour who was 3 feet away has become 6 feet away; the man over yonder who was 20 feet away is now 40 feet away. Each has moved proportionately to his distance from you, which is precisely what the spiral nebulæ are observed to be doing. The motion is not directed from any one centre, but is a general expansion, such that each individual observes every other individual to be receding.

In 1917, before any hint of this phenomenon had been obtained from observation, Prof. W. de Sitter was on the look out for something of the kind. He found that, on one of two alternative hypotheses arising out of Einstein's relativity theory, the light of very remote objects should be displaced to the red as though they were moving away from us; and he suggested the observed motions of the spiral nebulæ (by far the most remote objects known) as a discriminating test. At that time, only three radial velocities had been published, and they rather lamely supported his hypothesis by a majority of 2 to 1 . The majority has now become about 85 to 5 , and the five exceptions are nebulæ close to us which in any case should have had only small receding velocities. De Sitter's theory has been developed and modified by Friedman and Lemaître; the modern view of it is as follows :

Einstein's law of gravitation contains a term called the 'cosmical term' which is extremely small in ordinary applications to the solar system, etc., and is generally neglected. The term, however, actually represents a repulsive force directly proportional to the distance; so that however small it may be in ordinary applications, if we go

* Friday evening discourse delivered at the Royal Institution on Jan. 22.

No. 3255, VoL. 129] to distances sufficiently great it must ultimately become important. It is this cosmical repulsion which is, we believe, the cause of the expansion of the great system of the nebulæ. The repulsion may be to some extent counterbalanced by the ordinary gravitational attraction of the nebulæ on one another. This countervailing attraction will become weaker as the expansion increases and the nebulæ become farther apart. It seems likely that the universe started with a balance between gravitational attraction and cosmical repulsion; this equilibrium state is called an 'Einstein universe'. But it can be shown that the Einstein universe is unstable; and the slightest disturbance will cause either the repulsion or the gravitation to gain the upper hand, so as to topple the system into a state of continually increasing expansion or continually increasing contraction. Apparently, expansion won the initial struggle, and as the nebulæ spread apart, the opposition of gravitation became less and less, until now it is comparatively insignificant.

We see, then, that according to observation the system of the spiral nebulæ is expanding, and that relativity theory had foreseen just such an expansion (except that as an alternative it would have been content with an equally regular contraction). What better agreement could we desire? Nevertheless, there were some misgivings which I would not by any means condemn as unreasonable. It is true that theory predicted an effect of the kind observed, but it did not say how rapid the expansion would be. It expressed it in terms of an unknown ' cosmical constant' $\lambda$, leaving $\lambda$ to be determined by observation. Now the rate of expansion indicated by observation comes to us as a great shock. The universe is expanding so as to double its dimensions every 1300 million years ; that is no more than the period of geological time. Astronomers, who had been picturing a slow evolution of the stars extending over billions of years, would scarcely believe our staid old universe capable of such a hustle. In fact, it means a cut of something like ninety-nine per cent in our timescale, which even in these days of economy cuts is not to be accepted lightly by the department concerned. For this reason many have thought that the receding motions of the spiral nebulæ cannot be accepted as genuine, and that the whole phenomenon must be explained away as a misinterpretation of the red-shift observed in their spectra.

I think, however, that we shall have to accept the expansion. My reason is that it now seems possible to calculate the cosmical constant $\lambda$ by pure physical theory. The value is the same as that given by the recession of the nebulæ, so that there is full confirmation.

I have been tracing the effects of the cosmical constant in the behaviour of the great system of galaxies-phenomena on the grandest scale we 
have yet imagined. Now I want to turn to the other end of the scale and look into the interior of the atom, where, I think, we shall find that the same cosmical constant turns up again. It is, in fact, the main key to the mystery of protons and electrons. I cannot go very far into this part of the theory, but I will try to show why I am convinced that the cosmical constant comes into the theory of the atom. I must premise one thing. It is well known that, in Einstein's theory, gravitation has an interpretation not only as a force but also as a geometrical property - a curvature - of spacetime. So also the cosmical constant has an interpretation not only in terms of repulsive force but also as a measure of curvature. The constant $\lambda$ is, in fact, precisely equal to $1 / R^{2}, R$ being the radius of the world in the equilibrium (Einstein) state from which we suppose it to have started.

Length is necessarily relative. That is one of the results of Einstein's theory which has become almost a commonplace of physics; but it was a rather complicated kind of relativity that Einstein considered-relativity to the motion of our frame of reference. I am going to refer to another much more elementary relativity of length, namely, that length always implies comparison with a standard of length. It is only the ratio of lengths that enters into our experience. Suppose that every length and every distance in the universe were suddenly to be doubled; nothing would seem altered. I do not think we could attach any meaning to the change. Intrinsically, Brobdingnag and Lilliput are precisely the same; it needs an intruding Gulliver-an extraneous standard of length-to make them appear different.

Now, it is commonly stated in physics that all normal hydrogen atoms have the same size, or have the same spread of electric charge. We have a very fundamental equation (the wave equation) determining the spread, which is supposed to apply to any hydrogen atom and, of course, gives the same result for all. But what do we mean by their having the same size? Or it may be better to put the question negatively-What would it mean if we said that two hydrogen atoms were of different sizes, that is, similarly constructed but on different scales? It would be Brobdingnag and Lilliput over again. To give any meaning to the difference, we need a Gulliver. Now, the Gulliver of physics is always supposed to be a certain bar of metal called the International Metre. He is anything but a traveller; I think he has never been away from Paris. It was Prof. Weyl who first directed attention to the very big hiatus involved, when we speak of a length such as the radius of a hydrogen atom being a certain fraction of the standard metre. We have, as it were, our Gulliver but have left out his travels. The travels are (as Weyl showed) the interesting part of the story, and are not to be glossed over as irrelevant.

Weyl went further and pointed out that there is a natural standard of comparison which is always on the spot, namely, the radius of curvature of the world at that spot. We can thus give a direct meaning to the statement that two hydrogen atoms in any part of the universe have the same size; we mean that each of them is the same fraction of the radius of curvature of space-time at the place where it lies. The atom here is a particular fraction of the radius of curvature here; the atom on Sirius is the same fraction of the radius of curvature at Sirius. Whether the radius of curvature here is the same as at Sirius does not arise, and I do not think there is any meaning in trying to compare them.

The above definition of equality, and the use of Weyl's standard, may seem a dangerous innovation; but, indirectly, we have been using it all along, without knowing that we were doing so. Some years ago I pointed out that Einstein's law of gravitation can be stated in the form, "What we call a metre at any place and in any direction is a constant fraction of the radius of curvature of space-time for that place and direction". That is simply a translation of the law from symbols into words. The law is verified by observation, so that the statement gives us not only an ideal definition of the metre but also one which we know will accord with the reckoning of metres that is actually used. Thus, measurement in terms of the metre is equivalent to measurement in terms of the world radius, since the two standards are always in a constant ratio. Practically, it is more convenient to employ the metre, but in pursuing the theory we must go direct to the world radius; for obviously a particular bar of metal at Paris can have no fundamental status in physics and is altogether irrelevant to equations describing the mechanism of the atom. The world curvature, on the other hand, is on the spot and is directly reacting with the atom.

I now return to the wave equation which professes to determine how large an atom will be. That, as we have seen, means that it finds the ratio of the various intervals in the atom to the world radius there; so the world radius must come into the equation. But the world radius is the cosmical constant in another form. The cosmical constant has cropped up again inside the atom.

My task now was to spot the cosmical constant or the world radius in the current form of the wave equation, which is known by experiment to be substantially correct. It is very much disguised, because the current equation introduces the standard metre and all sorts of irrelevancies. But one knows the sort of effect that curvature can have; and the way it will appear in the equation is pretty well dictated by the quantum laws, which make a speciality of the properties of ' closed circuits' such as are introduced by curved space. I think I succeeded, and I arrived at the identification

$$
m c^{2} / e^{2}=\sqrt{ } N / R .
$$

The left side is a term in the current wave equation, and its value is known experimentally. The right side is the way that we write it now that we have penetrated its disguise. $R$ is the Einstein radius of the world, equal to the inverse square root of the cosmical constant; $N$ is the number of electrons (or protons) in the universe.

No. 3255, VoL. 129] 
This additional equation, combined with other equations already known, gives all the information required. We deduce, for example, that the number of electrons in the universe is $1 \cdot 29 \times 10^{79}$; and that the original radius of the universe, before it started to expand, was 1070 million light-years. Most important of all, we find that the consequent rate of expansion of the universe is $528 \mathrm{~km}$. per sec. per megaparsec distance. The observational determinations from the recession of the spiral nebulæ (which might be a little lower, since they include any countervailing gravitational attraction) range from $430 \mathrm{~km}$. to $550 \mathrm{~km}$. per sec. per megaparsec. We can feel little doubt, therefore, that the observed motions of the nebulæ are genuine and represent the expansion effect predicted by relativity. We must reconcile ourselves to this alarming rate of expansion, which plays havoc with older ideas as to the time-scale.

However interesting may be the application of this theory to the universe, the application to the interior of the atom seems likely to be still more fruitful. Now that we know the magnitude of the radius of curvature, we can set aside the arbitrary metre and use this natural unit in our equations. The big uninformative coefficients disappear ; and the equations are so much simplified that, I think, I have a fair idea of what they really mean and how they work. In particular, the relation of the proton to the electron is now apparent, and the theoretical ratio of their masses is found to be $1847 \cdot 6$; this is certainly very near to the observed value.

I do not want to stress too much the accuracy or finality of these fir $t$ results. I cannot see how anything can possibly be wrong with them; but then one never does see these faults until some new circumstance arises or some ingenious person comes forward to show us how blind we have been. At least, a way of progress has been found. I think that some day, when electrons and protons have come to order, we shall look back and see that the key to the mystery was lying somewhere in intergalactic space and was picked up by astronomers who measured the velocities and distances of nebulæ ten million light-years away.

\section{Goethe as Biologist}

\section{By Prof. F. J. CoLE, F.R.S., University of Reading}

$\mathrm{T}$ HE celebration of the centenary of the death of Goethe, which occurred on March 22, 1832, has evoked a series of works dealing with the activities of that unique genius. Among them is a critical biography by Prof. J. G. Robertson, the eminent authority on the life and works of Goethe.* This treatise is outside the scope, but not indeed outside the interests, of a scientific journal such as NATURE, except for the fact that it includes an enlightening chapter on Goethe's contributions to science. As a matter of personal history Prof. Robertson can scarcely be expected to take other than a detached view of Goethe's scientific achievements, and it is therefore not surprising to find him stating that "we look to Goethe, not for scientific discovery, an activity with which many other minds were as able-and perhaps better able-to cope successfully, but to more precious discoveries in the realm of the spirit and the imagination. May we not thus cherish something of a grudge that his immersion in scientific pursuits took up so very large a share in his life?" Prof. Robertson, however, would be the first to admit that Goethe himself would have indignantly denounced such an attitude, which strikes at the root of one of his characteristic speculations as to the nature of ereative art. To him the living organism was a work of art which only an artist could be expected to comprehend. Further, it is precisely those attributes of the spirit and imagination which give to his scientific work the qualities we all find so fascinating.

It was in an essay written in 1795 , but not published until 1820, that Goethe focused attention on the unity of plan or organic constant which was

* "The lite and Work of Goethe, 1749-1832," By Prof. J. G. Robertson. P'p. xii $+350+-8$ plates. (London: Creorge Routledge and Sons, Ltd., 1932.) 12s. 6it. net. supposed to underlie the organisation of all living beings. Since there was only one plan or idea, the world was regarded as something akin to a vast musical symposium, in which the poverty of a solitary theme was enriched by an endless and expanding series of variations. All forms were interpreted in terms of this ideal simulacrum, $\dagger$ and even man himself must be studied comparatively, working downwards from higher to lower types. This is the so-called law of reduction, or, to paraphrase the words of Geoffroy-there is but a single intangible being which becomes patent to our senses under diverse forms. Goethe was, of course, not the first to adopt such an attitude, but he was the first to convert it into an ordered scientific theory. The plan was put into operation or integrated by a controlling dynamic principle or Bildungstrieb. It is obvious at the outset that the theory is a scheme of development and not a principle of evolution, and, further, that its working out must depend on the institution of a system of homologies. There is one attractive feature in this point of view. However wrong it may be, it attempts to provide a common explanation of organic phenomena, and does not countenance the artificial separation of animals and plants.

Goethe's eager pursuit of homologies, of uniformity in diversity, was bound to lead to the conception that there was only one animal. Not merely was there a single animal type, but the integration of the type itself was effected by linking up into a series a number of similar, complete, subordinate entities like a string of beads. He extended the same speculation to plants, and interpreted, as others had done before him, the

$\uparrow$ Goethe would not have amproved this term, but the 'reality' of the idea is that of a mental abstraction projected on to paper like Owen's Archetype. 